\title{
Cooperation and Routing in Multi-Hop Networks
}

\author{
Elzbieta Beres and Raviraj Adve \\ Dept. of Elec. and Comp. Eng. University of Toronto \\ 10 King's College Road \\ Toronto, ON M5S 3G4, Canada \\ email: \{eberes, rsadve\}ecomm.utoronto.ca
}

\begin{abstract}
We study the cross-layer problem of combining routing and cooperative diversity in multi-hop, bandwidthconstrained, networks with dedicated multiple access. Previous work in cooperative diversity nearly always assumes cooperation to be a positive. We show that in a large scale multi-hop network, cooperation must only be used selectively. Our figure of merit is achievable data rate between a source and destination at a fixed probability of outage. We show that enforcing multiple hops is detrimental to performance, since each extra hop requires bandwidth expansion. This performance can be significantly improved by incorporating a selective cooperative diversity scheme on a one-hop link. On the other hand, the simulation results show that cooperative diversity does not improve performance over a dynamic routing protocol which searches for the optimal, non-diversity, route. Including the search for cooperative nodes into the dynamic route search, however, does further increase flow rates by decreasing the average number of hops and thus decreasing the required bandwidth expansion. This paper therefore points to the importance of an integrated approach to routing and the physical layer in cooperative networks.
\end{abstract}

\section{INTRODUCTION}

Cooperative diversity has been shown to provide significant performance gains in wireless networks where communication is impeded by channel fading. Traditionally, cooperative diversity has been seen as extension to multi-antenna diversity systems, where maximal ratio combining (MRC) is the preferred method of implementing receive diversity. Recently, however, Selection Diversity, referred to as Opportunistic Relaying in [1] and Selection Cooperation in [2], has been shown to have some significant advantages over traditional MRC schemes. Because Selection Cooperation requires that the relay node first decode the source message, in a multi-hop setting, selection can be interpreted as a version of routing according to instantaneous channel conditions. Within an individual hop, selection cooperation differs from optimal routing only in the presence of a source-destination link (which provides for one additional diversity path). Routing and the implementation of cooperative diversity is, therefore, an inherently linked crosslayer problem that should not isolated for classification into network and physical layers. This point is also emphasized in [3], [4], which investigate channel-adaptive routing and propose a per-hop routing approach that maximizes their measure of information efficiency.

On a different front, there is increasing consensus in the research community that a cross-layer, channel-adaptive approach to routing is necessary for the continual evolution of networks. This is particularly true for static networks where channels change slowly, and such channel adaptation is possible. In random access networks, this problem is addressed in [5]-[9], where the channel aware routing also considers interference and multiple access. In [10], the authors approach this problem in an energy-limited, bandwidth-unlimited scenario. Similarly, Haenggi [11] analyzes various routing strategies under Rayleigh fading conditions and energy constraints. He argues that when various practical network and physical layer issues are considered, long hops are often preferable to short hops.

The problem of combining routing with cooperative diversity, however, has received very little attention. Boyer et al. [12] analyzed the bit error rate and diversity of multihop networks while considering power but not bandwidth constraints. To the best of our knowledge, all other research in this area has been in the sensor and ad-hoc community, where energy constraints dominate network performance. In [13][18], the energy consumption of the networks is minimized; only in [19] is the throughput maximized, but again under energy constraints.

While concentrating on energy issues, the authors of these papers either ignore bandwidth expansion due to the halfduplex constraint, or assume the availability of exact channel knowledge at the transmitting cooperative nodes that allows them to precode data and not suffer bandwidth expansion. Whereas this approach is appropriate for networks with battery-powered nodes, we focus here on static mesh networks which impose constraints very different from those in sensor or ad-hoc networks. Because nodes are static and attached to a fixed power supply, energy is no longer a scarce commodity, and is only an issue in so far as it influences the transmission of other nodes. On the other hand, these networks are planned for transmitting high-throughput data, a fact which motivates a design which maximizes the transmission rate. Furthermore, as argued in [20], as these networks mature, they will enter a period of throughput growth characterized by a scarcity of bandwidth, a resource which cannot be neglected.

To address these issues, we focus on the problem of combining routing and cooperative diversity in a multi-hop mesh network with static nodes. We frame this problem as a rate-maximization problem in a bandwidth-limited, effectively energy-unlimited regime. We use a dedicated multiple-access scheme such as TDMA to resolve the issue of poor scalability of random access protocols [21]. This also allows us to eliminate the MAC layer from our analysis, hence simplifying 
the problem at hand. Because cooperation imposes a causality structure, TDMA is also particularly well suited, and often considered, for systems employing cooperation [22].

We implement cooperative diversity using Selection Cooperation; we note, however, that the results should hold for any cooperative scheme. As our results will show, cooperative diversity does not provide any rate gains over an optimal routing algorithm. This fact is also commented on in [23]. However, if cooperative diversity is incorporated into the route selection algorithm, the number of required hops decreases and significant gains are possible. A simple, selective implementation of cooperative diversity into one-hop systems has comparable performance to the more complex routing algorithms. This paper therefore underscores the importance of integrating routing and the selective use of cooperative diversity. An issue not answered in this paper is whether such an integrated optimal algorithm is feasible in real time. This paper develops performance bounds; it does not attempt to propose a new routing algorithm.

This paper is structured as follows. Section II describes the system model. Section III describes the routing and cooperative diversity schemes considered in the paper, and the results of simulations evaluating these schemes are presented in section IV. Section V concludes the work.

\section{SySTEM MODEL}

In this section, we discuss the network layout, channel model, the the multiple access scheme, and the constraints applied throughout the paper.

\section{A. Network Model}

We consider a distributed system, as shown in Figure 1, consisting of a source $S$, destination $D$, and $N M$ stationary nodes aligned in a uniformly placed grid. The distance between a node and its four nearest neighbors is $d$. This simple structure models mesh and other networks where nodes are static with known locations.

The channel between nodes in locations $(i, j)$ and $(k, l)$, $h_{(i, j)-(k, l))}$, is modelled as flat and slowly-fading Rayleigh with variance $1 / \lambda_{(i, j)-(k, l)} ;\left|h_{(i, j)-(k, l))}\right|^{2}$ is exponential with parameter $\lambda_{(i, j)-(k, l)}$. This channel is independent of all other channels between remaining nodes. $\lambda_{(i, j)-(k, l)}$, which is inversely proposal to the average channel power, is a function of inter-node distance, $d_{(i, j)-(k, l)}$, through the attenuation exponent $p$, i.e., $\lambda_{(i, j)-(k, l)} \propto d_{(i, j)-(k, l)}^{p}$. We do not include shadowing into the fading model, although this can easily be incorporated. Note that assuming static nodes and very slowly-fading channels is crucial to the discussion of routing protocols which adapt to changing channels conditions. Such an adaptation is only possible if the channels are changing slower than the possible rate of adaptation.

A source node $S$ transmits data to destination node $D$ with the help of the $N M$ nodes in the grid. The data can be routed from the source to destination over multiple hops, and the nodes may use cooperative diversity within each hop. We denote the communication between the source and destination

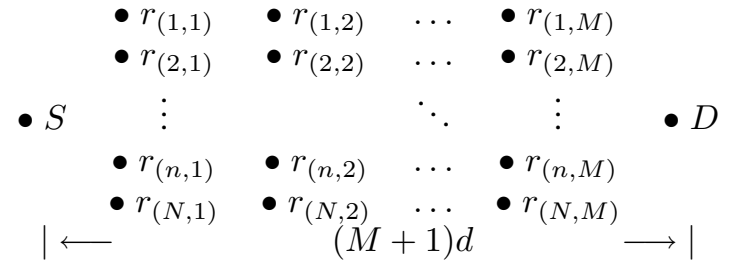

Fig. 1. $\quad N \times M$ Network Layout. $r_{(x, y)}$ denotes a node in position $(x, y)$. The horizontal and vertical distance between each node is $d$.

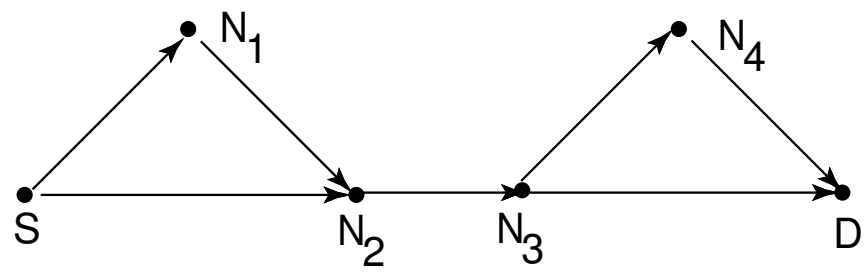

Fig. 2. Illustrating multi-hop communications with cooperative diversity.

as a flow, and each hop between intermediary nodes as one hop. Figure 2 illustrates a flow over three hops; cooperative diversity is used in the first and third hops only. Note that this optional use of cooperation is unlike other network models in the available literature.

\section{B. Multiple Access}

As discussed in the introduction, we consider a dedicated MAC protocol such as TDMA. The source uses a total of $W$ degrees of freedom (possibly bandwidth in $\mathrm{Hz}$ ) to transmit data to the destination. The half-duplex constraint precludes nodes from transmitting on the same channel simultaneously; in a flow with $N_{h}$ hops, therefore, each hop uses $\left(W / N_{h}\right)$ degrees of freedom. This model explicitly accounts for the increased traffic due to splitting large hops or implementing cooperative diversity. Although it is be possible to optimize the degrees of freedom allocated to each hop, this analysis is beyond the scope of this paper.

Because the nodes considered in this paper are stationary and attached to a fixed power supply, their life-time is not a consideration. Individual power is therefore not constrained and each node transmits at its maximum available power of $P$ Watts. Furthermore, the dedicated MAC protocol eliminates the need for strict power control, and the total power is also unconstrained: a flow through $N_{h}$ hops, for example, would consume $N_{h} P$ Watts. Although it might at first glance seem unfair to compare two scenarios with different hop numbers, each using different power levels, we argue that such a scenario is applicable to real systems with stationary nodes with dedicated power supplies.

\section{Routing AND COOPERATION AlgorithmS}

In this section, we discuss the various schemes used to transmit information from the source $S$ to the destination $D$. Note that most of these algorithms require knowledge of forward channel power, which requires either centralization 
or a scheme to distribute channel information throughout the network. We assume, however, that because the nodes are stationary, the channel is changing slowly enough to allow for such a distribution of information. At the same time, we stress that the algorithms discussed in this section serve as performance bounds only; their implementation would require the development of more efficient routing algorithms.

The channel between nodes $(i, j)$ and $(k, l),\left|h_{(i, j)-(k, l)}\right|$, implies a mutual information between the nodes, $I_{(i, j)-(k, l)}=$ $\log \left(1+\left|h_{(i, j)-(k, l)}\right|^{2}\right)$. To be sustainable, the data rate over this channel, $R_{(i, j)-(k, l)}$ must be less than the mutual information.

\section{A. 1-Hop Smart Cooperation}

The 1-Hop Smart Cooperation algorithm optionally implements cooperative diversity without routing. Motivated by [2], we consider Selection Cooperation and always choose at most one "best" cooperative partner. Unlike the scheme in [2], however, the Smart Cooperation algorithm implements cooperation only if it is advantageous in terms of rate. The destination coherently combines the two transmissions.

Without cooperation, the mutual information between the source and destination is simply

$$
I_{n o-\text { coop }}=\log \left(1+\left|h_{S D}\right|^{2}\right) .
$$

Consider a decode-and-forward cooperative diversity scheme and a single cooperative node, $r_{(i, j)}$. Given a strong $S-$ $r_{(i, j)}$ channel, the cooperative mutual information between the source and destination with $r_{(i, j)}$ acting as a relay is given by

$$
I_{\operatorname{coop}_{(i, j)}}=\frac{1}{2} \log \left(1+\left|h_{r_{(i, j)} D}\right|^{2}+\left|h_{S D}\right|^{2}\right),
$$

where the pre-log factor of (1/2) indicates the further halving required to implement cooperative diversity with a half-duplex constraint. To prevent error propagation, node $r_{(i, j)}$ can only relay if it has correctly received the message from the source,

$$
I_{s r_{(i, j)}}=\frac{1}{2} \log \left(1+\left|h_{S r_{(i, j)}}\right|^{2}\right)>I_{\operatorname{coop}_{(i, j)}} \text {. }
$$

Denote as the decoding set of $S, \mathcal{D}(s)$, as the set of all cooperative nodes satisfying (3). The relay chosen from this set maximizes the cooperative mutual information, i.e.,

$$
I_{\text {coop }}=\max _{(i, j) \in \mathcal{D}(s)} I_{\operatorname{coop}_{(i, j)}} .
$$

To ensure that cooperation always helps, the source uses cooperative diversity only if it increases the achievable rate. The final rate of the Smart Cooperation algorithm, $R_{S C-1}$, is therefore the maximum between the cooperative and noncooperative mutual information:

$$
R_{S C-1}=\max \left\{I_{\text {coop }}, I_{\text {non-coop }}\right\} \text {. }
$$

\section{B. 1-Hop Simple Cooperation}

The Simple Cooperation scheme differs from the Smart Cooperation scheme only in the last step. Whereas in Smart Cooperation the relay is active only if cooperation improves the rate, in Simple Cooperation the relay is always active. This is the traditional model for cooperative diversity. The resulting rate is simply the cooperative mutual information: $R_{S i C-1}=I_{\text {coop }}$. Clearly, $R_{S i C-1} \leq R_{S C-1}$. The question is how often does the increased signal-to-noise ratio (SNR) due to maximal ratio combining outweigh the pre-log factor of $(1 / 2)$ ?

\section{2-Hop Smart Cooperation}

The 2-Hop Smart Cooperation algorithm is very similar to the 1-Hop Smart Cooperation algorithm discussed above, with the exception that the algorithm forces two hops. This scheme is the simplest from those combining Smart Cooperation and multi-hop communications. The source transmits to the intermediary node $n_{I}$ with index $\left(\left\lfloor\frac{N}{2}\right\rfloor,\left\lfloor\frac{M}{2}\right\rfloor\right)$, where $\lfloor\cdot\rfloor$ indicates rounding to the nearest lower integer. 2-Hop Smart Cooperation is implemented to increase the rate $R_{S-n_{I}}$ between the source and $n_{I}$, and the rate $R_{n_{I}-D}$ between $n_{I}$ and the destination. For both hops, all $\frac{N M}{2}-1$ are used as potential cooperative nodes (the intermediary node is precluded from cooperating with itself). The final rate, $R_{S C-2}$ is the minimum of the rates achieved on both hops, $\min \left\{R_{S-n_{I}}, R_{n_{I}-D}\right\}$. The two hop transmission implies that only $W / 2$ degrees of freedom are available for each hop.

\section{Dynamic Routing}

Dynamic routing searches for the rate-maximizing path between the source and destination. To ensure forward progress, we constrain the maximum number of hops to $M+1$, i.e., there can never be hopping backwards or along the same vertical axis. The rate $R_{D R}$ is the maximum of all rates achieved using any number of hops less than $M+1$. For a specific $N_{h}$-hop flow, the flow rate is $\left(1 / N_{h}\right)$-th of the minimum of the mutual information achieved on each hop. As discussed in Section II-B, the $\left(1 / N_{h}\right)$ factor accounts for the half-duplex constraint and penalizes multiple-hop routes. The algorithm can be summarized as follows:

1) Determine the one-hop rate: $R_{1}=I_{S D}$.

2) For $2 \leq N_{h} \leq(M+1)$, determine the rate achieved on each of the possible $N_{h}$-hop flows. This rate is the maximum of the minimum of the $N_{h}$ mutual information terms:

$$
\begin{aligned}
R_{N_{h}} & =\max \min \frac{1}{N_{h}}\left\{I_{S-(i, j)}, I_{(i, j)-(k, l)}, \ldots, I_{(m, n)-D}\right\} \\
& 1 \leq(i, k, \ldots m) \leq N, 1 \leq j<l, \ldots<n \leq M .
\end{aligned}
$$

For each $N_{h}$, the maximization is over all possible flows consisting of $N_{h}$ hops, and thus over all node-indices. The strict inequality in the $y$-coordinate ensures forward progress by eliminating the possibility of routing backwards or along a vertical line (note that in our definition, the $S-D$ link is along the $y$-direction).

3) Find the maximum over all the flows:

$$
R_{D R}=\max \left\{R_{1}, R_{2}, \ldots, R_{M+1}\right\} .
$$

\section{E. Dynamic Routing with Cooperation}

The Dynamic Routing with Cooperation algorithm sequentially combines Dynamic Routing and Smart Cooperation. The 
optimal dynamic route is chosen using the Dynamic Routing algorithm. Given this route, cooperation is used to increase the rate along this path, i.e., Smart Cooperation is applied between the nodes chosen by the Dynamic Routing algorithm.

\section{F. Dynamic Cooperative Routing}

Dynamic Cooperative Routing simultaneously combines Dynamic Routing with Cooperation: the optimal path is chosen together with the cooperative partners. As in Dynamic Routing, the Dynamic Cooperative Rate $R_{D C R}$ is the maximum of the rates achieved with different hops:

$$
R_{D C R}=\max \left\{R_{1}, R_{2}, \ldots, R_{M+1}\right\} .
$$

Unlike Dynamic Routing, however, $R_{D C R}$ is achieved with cooperation potentially included in each of the $N_{h}$ hops, i.e., for each possible combination of hops, the algorithm implements Smart Cooperation along each hop. With the exception of the cooperation included in the search for optimal route, this algorithm is very similar to Dynamic Routing. In the interest brevity, we provide only an outline of this algorithm below:

1) Perform the Smart Cooperation algorithm. The result is $R_{1}$, the maximum rate achieved with one hop.

2) For $N_{h} \leq M+1$, find a set of all possible $N_{h}$-hop routes between source and destination. For each route, perform Smart Cooperation along all hops, i.e., determine if any cooperating partner can increase the rate along any of the $N_{h}$ hops. $R_{N_{h}}$, the rate of a $N_{h}$-hop route, is the minimum rate along each of the $N_{h}$ hops (including a factor of $1 / N_{h}$ for bandwidth expansion).

3) The final rate $R_{D C R}$ is the maximum of the rates obtained using all possible routes and cooperating partners.

Note that dynamic cooperative routing is an extremely complex scheme with large computation overhead.

In summary, the routing and cooperative schemes considered include, in increasing order of complexity, the traditional model for cooperative diversity, Smart Cooperation, cooperation within multi-hop communications, and also "optimal" schemes such as dynamic routing (without cooperation) and the combination of dynamic routing with cooperation (in both sequential and simultaneous flavors).

\section{Results}

In this section, we present results of the schemes discussed in Section III. A mathematical analysis of these schemes is highly complicated by the necessity to make high SNR approximations which do not hold for medium SNRs and higher rates. Although there has been some effort to find good approximations to the outage probability of cooperative diversity systems [24], these approaches are insufficient for the complex routing protocols considered here. Even the analysis for the Smart Cooperation algorithm, which requires no routing, appears intractable because of the influence of the source-relay channel on the final rate. For the purposes of this paper, therefore, we present simulation results only, while

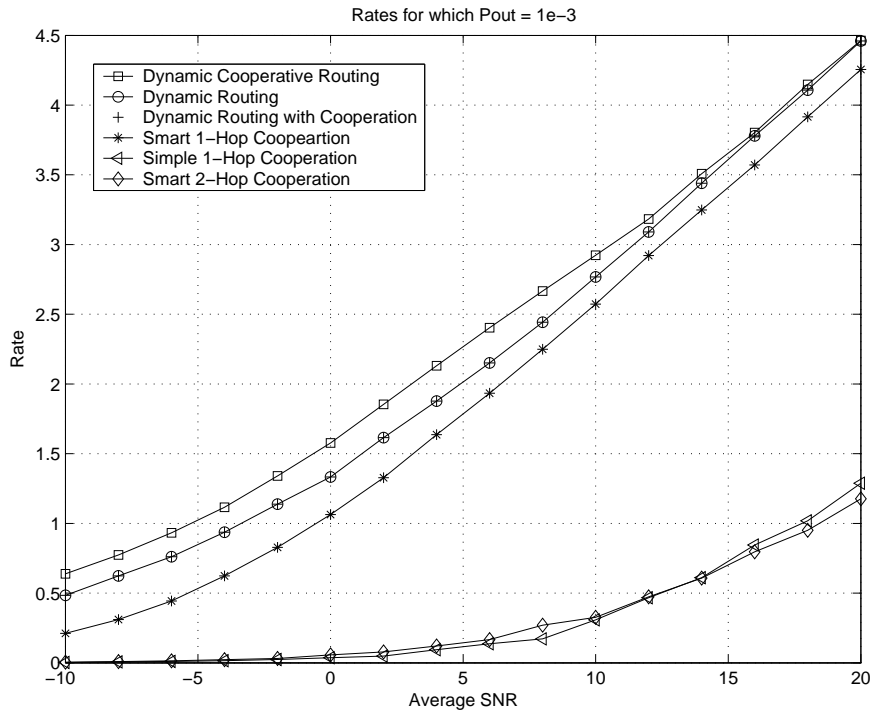

Fig. 3. Rate obtained for $P_{\text {out }}=10^{-3}$ and $N=M=9$.

continuing to search for approximations which can simplify the underlying analysis of the schemes.

In the simulations, we choose a $9 \times 9$ network (i.e., $N=$ $M=9$ ) and an attenuation exponent of $p=2.5$. This choice is motivated by the scenario of planned static meshnodes installed on posts; transmissions between such nodes should undergo little shadowing effects and a lower attenuation exponent.

The results presented are averaged obtained from 60000 fading realizations, and the resulting cumulative density function, $F_{R}(r)$, of the instantaneous rate, calculated according to the methods described in Section III. The rate presented for a specific SNR is that for which the probability of outage is $10^{-3}$, i.e., $F_{R}^{-1}\left(10^{-3}\right)$.

The main results are displayed in Figure 3. The rates, as defined in the above section, are displayed as a function of receive SNR at the destination. This model was chosen to simplify fair comparisons between various network sizes, as it is independent of the distances between the nodes. This figure is thus the central contribution of this paper. To gain intuition about the behavior of the algorithms, we simulate their performance for SNRs as high as $20 \mathrm{~dB}$. We note, however, that for any reasonable distances between the nodes, such high receive SNR levels would be highly unlikely.

From Fig. 3, several interesting conclusions can be drawn. The very poor results of the 2-hop Smart Cooperation scheme of Section III-C demonstrate the loss of performance of any routing algorithm that makes routing decisions "off-line". The poor performance of this scheme is due to bandwidth expansion: even for a high instantaneous SNR, where it might be advantageous to route directly to the destination and not undergo the bandwidth penalty of splitting the transmission in two, the scheme forces the two hops and always undergoes this penalty. Similarly, always enforcing cooperation, as in the simple 1-hop cooperation scheme of Section III-B, also 


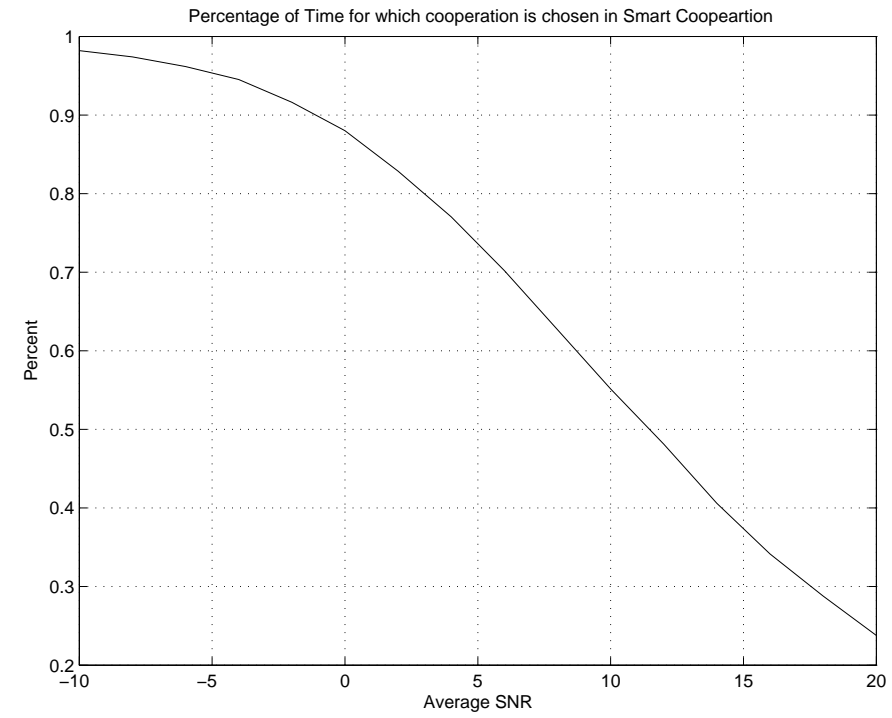

Fig. 4. Percentage of time cooperation occurs when Smart Cooperation is implemented. $P_{\text {out }}=10^{-3}$ and $N=M=9$.

provides poor performance.

The following conclusion is an optimistic one: focusing on the single hop with smart cooperation scheme of Section IIIA, even without a routing protocol and a with direct transmission between the source and destination, cooperative diversity significantly improves the flow rate. Thus with minimal complexity, very good performance can be obtained with a simple 1-hop scheme and a Smart Cooperation diversity scheme. The results of the 1-hop Simple Cooperation scheme, however, indicate the importance of cooperating only when necessary, an observation verified in Fig. 4. Here, we plot the percentage of hops which use cooperation (in the Smart Cooperation scheme) as a function in SNR. As the SNR increases, the use of cooperation becomes more sporadic and the direct source-destination link becomes advantageous to a cooperative approach.

Returning to Fig. 3, the 1-Hop Smart Cooperation scheme is outperformed by the Dynamic Routing algorithm which always chooses the best non-cooperative route. This result is unsurprising, as this algorithm is more complex and requires more distributed knowledge or centralization. It is also important to realize that, although we have referred to this scheme as non-cooperative, it is not devoid of diversity: optimally selecting the best route ensures that data is always sent on high-SNR links, which is conceptually a diversity notion.

Perhaps more surprisingly, this algorithm has identical performance to one where cooperative diversity is applied directly on top of the predetermined, dynamically chosen route. This has interesting implications: cooperative diversity offers no benefit when a good path has already been chosen. Note that this is consistent with the work of [23]. However, this does not imply the cooperative diversity cannot help. If cooperation is included in the routing protocol figures of merit, as proposed in Section III-F, higher data rates are possible. This scheme

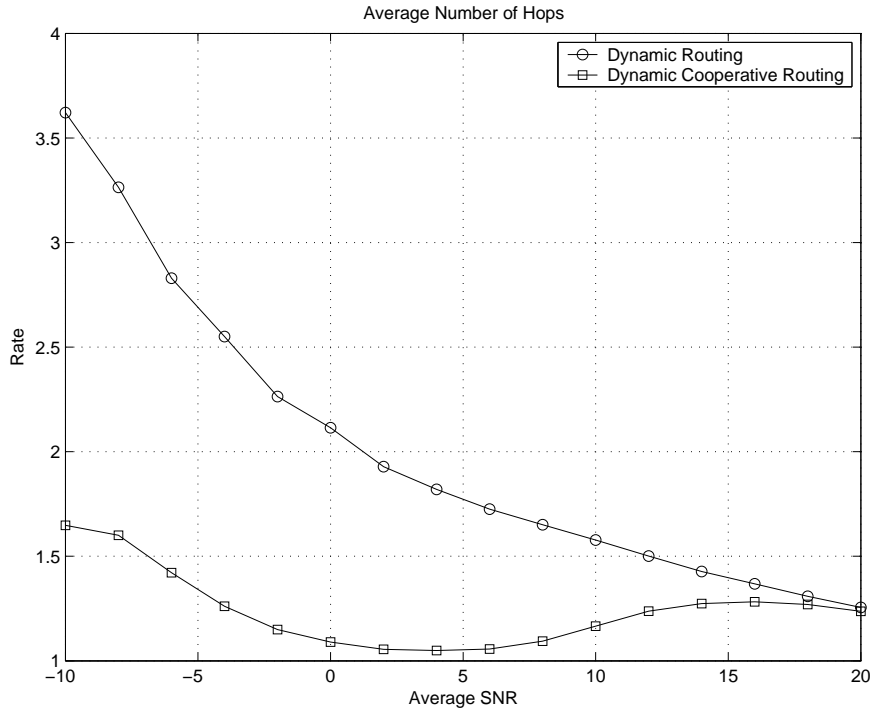

Fig. 5. Average number of hops obtained when $P_{\text {out }}=10^{-3}$ and $N=$ $M=9$.

corresponds to the curve marked "Dynamic Cooperative Routing" in Fig. 3.

The intuition for this can be obtained from Fig. 5, where we plot the average number of hops - averaged over all Monte Carlo runs - obtained with the Dynamic Routing (Section III-D) and Cooperative Dynamic Routing ((Section III-F) schemes. The performance improvement of the Cooperative Dynamic Routing scheme thus comes from the influence of the cooperative node which can, on average, encourage longer hops. Cooperative Dynamic Routing decreases the number of hops, thus decreasing bandwidth expansion. This also explains why cooperative diversity applied onto the predetermined dynamic routes offers no help: because the routes are predetermined, this method cannot decrease the number of hops.

\section{CONCLusions}

The available literature in cooperative diversity generally assumes cooperation to be a net positive. This is mainly because this body of work has focused on small networks with very few nodes. Network issues such as routing have generally been ignored. Consequently, we are motivated by such an analysis in mesh networks wherein data rate is a primary figure of merit. Given this lack of research into the interdependence between routing and cooperative diversity in such bandwidth-limited, power-unlimited networks, we have simulated several schemes which combine various degrees of optimality of routing and diversity implementation.

Although our simulations are based on Selection Cooperation, we expect these conclusions to hold for any cooperative scheme. The results point to a careful and discriminate approach to multi-hopping: in general, fewer hops are preferred, and a route should only be split if the high quality of the multi-hop channels can compensate for the loss of bandwidth penalty incurred from multi-hopping. Breaking routes into 
several hops off-line results in a dramatic performance loss in terms of rate; interestingly, cooperative diversity by itself, added onto the individual hops, does not compensate for this loss. Dynamically choosing routes according to changing channel conditions performs very well and, once again, applying cooperative diversity on the chosen nodes has no benefit. In this scenario, cooperative diversity is beneficial only if the search for the cooperating nodes is included into the dynamic routing algorithm; this benefit is due to longer hops and reduced bandwidth expansion. Cooperative diversity is also beneficial when applied with very little complexity to a one-hop system. As long as cooperation is used only when it can increase rate, the results are comparable to the more complex dynamic-routing schemes.

\section{REFERENCES}

[1] A. Bletsas, A. Khisti, D. Reed, and A. Lippman, "A simple cooperative diversity method based on network path selection," IEEE Journal on Selected Areas of Communication, vol. 24, pp. 659-672, March 2006.

[2] E. Beres and R. S. Adve, "On selection cooperation in distributed networks," in Proceedings of the 2006 Conference on Information Sciences and Systems (CISS 2006), March 2006.

[3] M. R. Souryal, B. R. Vojcic, and R. L. Pickholtz, "Information efficiency of multihop packet radio networks with channel-adaptive routing," IEEE Journal on Selected Areas in Communications, vol. 23, pp. 1553-1562, January 2005.

[4] M. R. Souryal and N. Moayeri, "Channel-adaptive relaying in mobile Ad Hoc networks with fading," in Proceedings of the 2005 Second Annual IEEE Communications Society Conference on Sensor and Ad Hoc Communications and Networks, 2005 (IEEE SECON 2005), September 2005.

[5] X.-H. Lin, Y.-K. Kwok, and V. Lau, "A quantitative comparison of Ad Hoc routing protocols with and without channel adaptation," IEEE Transactions on Mobile Computing, vol. 4, pp. 111- 128, March-April 2005.

[6] D. S. J. D. Couto, D. Aguayo, J. Bicket, and R. Morris, "A highthroughput path metric for multi-hop wireless routing," in Proceedings of ACM MobiCom, September 2003.

[7] R. Draves, J. Padhye, and B. Zill, "Routing in multi-radio, multi-hop wireless mesh networks," in Proceedings of ACM MobiCom, 2004), September 2004.

[8] L. Iannone and S. Fdida, "Evaluating a cross-layer approach for routing in wireless mesh networks," Telecommunication Systems Journal (Springer) Special issue: Next Generation Networks - Architectures, Protocols, Performance, vol. 31, pp. 173-193, March 2006.

[9] S. Biswas and R. Morris, "Opportunistic routing in multi-hop wireless networks," SIGCOMM Computer Communications Review, vol. 34, pp. 69-74, 2004.

[10] A. H. K. Seada, M. Zuniga and B. Krishnamachari, "Energy efficient forwarding strategies for geographic routing in wireless sensor networks," in Proceedings of ACM SenSys, November 2004.

[11] M. Haenggi, "On routing in random rayleigh fading networks," IEEE Transactions on Wireless Communications, vol. 4, pp. 1553-1562, July 2005.

[12] J. Boyer, D. Falconer, and H. Yanikomeroglu, "Multihop diversity in wireless relaying channels," IEEE Transactions on Communications, vol. 52, pp. 1820 - 1830, October 2004.

[13] S.-H. Chen, U. Mitra, and B. Krishnamachari, "Cooperative communication and routing over fading channels in wireless sensor network," in Proceedings of the IEEE International Conference on Wireless Networks, Communications, and Mobile Computing (WirelessCom), June 2005.

[14] X. Fang, T. Hui, Z. Ping, and Y. Ning, "Cooperative routing strategies in Ad Hoc networks," in Proceedings of the IEEE Vehicular Technology Conference (VTC-Spring 2005), June 2005.

[15] A. Khandani, J. Abounadi, E. Modiano, and L. Zhang, "Cooperative routing in wireless networks," in Proceedings of Allerton Conference on Communications, Control and Computing, October 2006.
[16] F. Li, A. Lippman, and K. Wu, "Minimum energy cooperative path routing in wireless networks: An integer programming formulation," in Proceedings of the 63th IEEE Vehicular Technology Conference (IEEE VTC '2006), May 2006.

[17] Y. Yuan, Z. He, and M. Chen, "Virtual MIMO-based cross-layer design for wireless sensor networks," IEEE Transactions on Vehicular Technology, vol. 55, pp. 856- 864, May 2006.

[18] Y. Yuan, M. Chen, and T. Kwon, "A novel cluster-based cooperative MIMO scheme for multi-hop wireless sensor networks," EURASIP Journal on Wireless Communications and Networking, vol. 2006, pp. Article ID 72493, 9 pages, 2006.

[19] V. Srinivasan, P. Nuggehalli, C.-F. Chiasserini, and R. Rao, "An analytical approach to the study of cooperation in wireless Ad Hoc networks," IEEE Transactions on Vehicular Technology, vol. 4, pp. 722 - 733, March 2005.

[20] M. Sikora, J. N. Laneman, M. Haenggi, D. J. C. Jr., and T. E. Fuja, "Bandwidth- and power-efficient routing in linear wireless networks," IEEE Transactions on Information Theory, vol. 52, pp. 2624 - 2633, June 2006.

[21] I. F. Akyildiz and X. Wang, "A survey on wireless mesh networks," IEEE Communications Magazine, vol. 43, pp. S23- S30, Sept 2005.

[22] J. N. Laneman, D. N. C. Tse, and G. W. Wornell, "Cooperative diversity in wireless networks: Efficient protocols and outage behavior," IEEE Transactions on Information Theory, vol. 50, pp. 3062 - 3080, December 2004.

[23] M. Qin and R. S. Blum, "Capacity of wireless Ad Hoc networks with cooperative diversity: a warning on the interaction of relaying and multihop routing," in Proceedings of the IEEE International Conference on Communications, (ICC 2005), May 2005.

[24] Y. Zhao, R. S. Adve, and T. J. Lim, "Outage probability at arbitrary SNR in cooperative diversity networks," IEEE Communication Letters, vol. 9, pp. 700-702, August 2005. 